\title{
Effects of comprehensive nursing intervention combined with respiratory functional exercises on pulmonary function and self-care ability in patients with pulmonary tuberculosis: results of a randomized trial
}

\author{
Zhaojia Xu ${ }^{1 \#}$, Wenwei Chen ${ }^{2 \#}$, Xiaosong $\mathrm{Li}^{3}$ \\ ${ }^{1}$ Intensive Care Unit, Wuhan Jinyintan Hospital, Wuhan, China; ${ }^{2}$ Department of Tuberculosis, Wuhan Jinyintan Hospital, Wuhan, China; \\ ${ }^{3}$ Department of Emergency, Wuhan Jinyintan Hospital, Wuhan, China \\ Contributions: (I) Conception and design: Z Xu; (II) Administrative support: X Li; (III) Provision of study materials or patients: Z Xu; (IV) Collection \\ and assembly of data: W Chen; (V) Data analysis and interpretation: X Li; (VI) Manuscript writing: All authors; (VII) Final approval of manuscript: \\ All authors. \\ "These authors contributed equally to this work. \\ Correspondence to: Xiaosong Li. Department of Emergency, Wuhan Jinyintan Hospital, No.1 Yintan Road, Dongxihu District, 430013, Wuhan, \\ China. Email: jip0870@163.com.
}

Background This study was carried out based on the background that pulmonary tuberculosis seriously threatens people's physical health while the current treatment has certain limitations.

Methods: A total of 150 patients with pulmonary tuberculosis who were admitted to Wuhan Jinyintan Hospital from January 2017 to December 2018 were selected as study participants. Patients were randomly divided into a control group $(n=75)$ who were treated with routine nursing, and an experimental group $(n=75)$ who were treated with comprehensive nursing intervention combined with respiratory functional exercises. Following treatment, the pulmonary function, self-care ability, and other indexes of the patients in both groups were compared and analyzed.

Results: After nursing intervention, the pulmonary function and self-care ability of patients in the experimental group were significantly higher than those in the control group. The scores of the 6-minute walking distance (6MWD) and modified Medical Research Council (mMRC) dyspnea scale of patients in both groups were significantly higher after nursing intervention compared to before intervention. Furthermore, after intervention, the scores in the experimental group were significantly higher than those in the control group. The partial pressure of oxygen $\left(\mathrm{PaO}_{2}\right)$, partial pressure of carbon dioxide $\left(\mathrm{PaCO}_{2}\right)$, and the quality of life of patients in the experimental group after intervention were significantly better than those in the control group.

Conclusions: Comprehensive nursing intervention combined with respiratory functional exercises can significantly improve the pulmonary function, self-care ability, and quality of life of patients with pulmonary tuberculosis, with obvious clinical efficacy.

Trial registration: Chinese Clinical Trial Registry ChiCTR2100048983.

Keywords: Comprehensive nursing; respiratory functional exercise; pulmonary tuberculosis; self-care ability; pulmonary function

Submitted Mar 31, 2021. Accepted for publication Jul 22, 2021.

doi: 10.21037/apm-21-1178

View this article at: https://dx.doi.org/10.21037/apm-21-1178

(c) Annals of Palliative Medicine. All rights reserved. 


\section{Introduction}

Pulmonary tuberculosis, also known as the white plague, is a chronic infectious disease caused by Mycobacterium tuberculosis and is mainly transmitted through the respiratory tract (1-4). According to the World Health Organization, pulmonary tuberculosis affects tens of millions of people worldwide each year, and the death toll can reach 3 million annually, making pulmonary tuberculosis one of the infectious diseases with the highest incidence and mortality worldwide, with China being one of the countries with the highest incidence. At present, medication is the top priority for the clinical treatment of pulmonary tuberculosis. However, long-term treatment with medication has its limitations including adverse reactions and difficulty in repairing damaged tissues (5-8). In recent years, respiratory functional nursing has been clinically recognized as a non-medicine therapy. It involves effective respiratory functional exercises which can increase body tolerance, relieve dyspnea, and improve immunity in patients $(9,10)$. We present the following article in accordance with the CONSORT reporting checklist (available at https://dx.doi.org/10.21037/apm-21-1178).

\section{Methods}

\section{General information}

This study was approved by the Ethics Committee of Wuhan Jinyintan Hospital (No.: 2016112505). All patients and their families were informed of the purpose and process of this study, received nursing intervention protocols, and signed the informed consent. The study followed the guild lines of Declaration of Helsinki (as revised in 2013) (11).

A total of 150 patients with pulmonary tuberculosis treated in our hospital from January 2017 to December 2018 were selected as study participants. Patients were randomly divided into a control group $(\mathrm{n}=75)$ and an experimental group $(\mathrm{n}=75)$ (Figure 1). The control group consisted of 44 males and 31 females, aged from 43 to 71 years, with an average age of $53.41 \pm 6.12$ years. The course of disease ranged from 1 week to 8 months. Within the control group, 59 patients had infiltrative pulmonary tuberculosis, 11 had tuberculous pleuritis, and 5 had hematogenous disseminated pulmonary tuberculosis. The experimental group consisted of 46 males and 29 females, aged from 42 to 72 years, with an average age of $54.55 \pm 6.23$ years. The course of disease ranged from 1 week to 7 months. Within the experimental group, 57 patients presented with infiltrative pulmonary tuberculosis,
12 had tuberculous pleuritis, and 6 had hematogenous disseminated pulmonary tuberculosis. These two groups were comparable, with no statistical differences in the general characteristics between the two groups $(\mathrm{P}>0.05)$.

\section{Inclusion and exclusion criteria}

Patients were included in this study if they met the following inclusion criteria: (I) clinical diagnosis of pulmonary tuberculosis; (II) the length of hospital stay was more than or equal to 2 weeks; and (III) complete clinical medical records were available.

Patients with the following conditions were excluded from this study: (I) tissue pathological changes in other organs such as brain, heart, kidneys, liver, etc.; (II) asthma, increased number of blood eosinophils, or other acute respiratory infectious diseases; and (III) mental and cognitive disorders or refusal to cooperate with the study.

\section{Study methods}

Patients in the control group received routine nursing of pulmonary tuberculosis. At admission to the hospital, nursing staff conducted an evaluation of the patient's physical condition, progression of the disease, and psychological changes. Specific routine nursing protocols were then formulated based on disease examination, mainly including monitoring changes in vital signs and disease condition, comforting patients and other nursing measures.

In the experimental group, patients were treated with comprehensive nursing intervention combined with respiratory functional exercise. Comprehensive nursing care involved numerous aspects. First, as with the control group, primary nurses evaluated patients and following advice from doctors, a comprehensive nursing protocol was designed. Psychological intervention involved detailed communication between medical staff and patients and their families to establish a good relationship with mutual trust. Staff should develop a comprehensive understanding of the patient's personality, characteristics, family background, mental status, and emotional changes. This will enable timely interventions to relieve negative emotions and enhance the patient's confidence in managing the disease. Additionally, trust and cooperation from the patient's family should also be encouraged (12-15). Health education on pulmonary tuberculosis was provided for patients and their families through group information sessions, individual guidance, one-to-one questions and answers, etc. through 


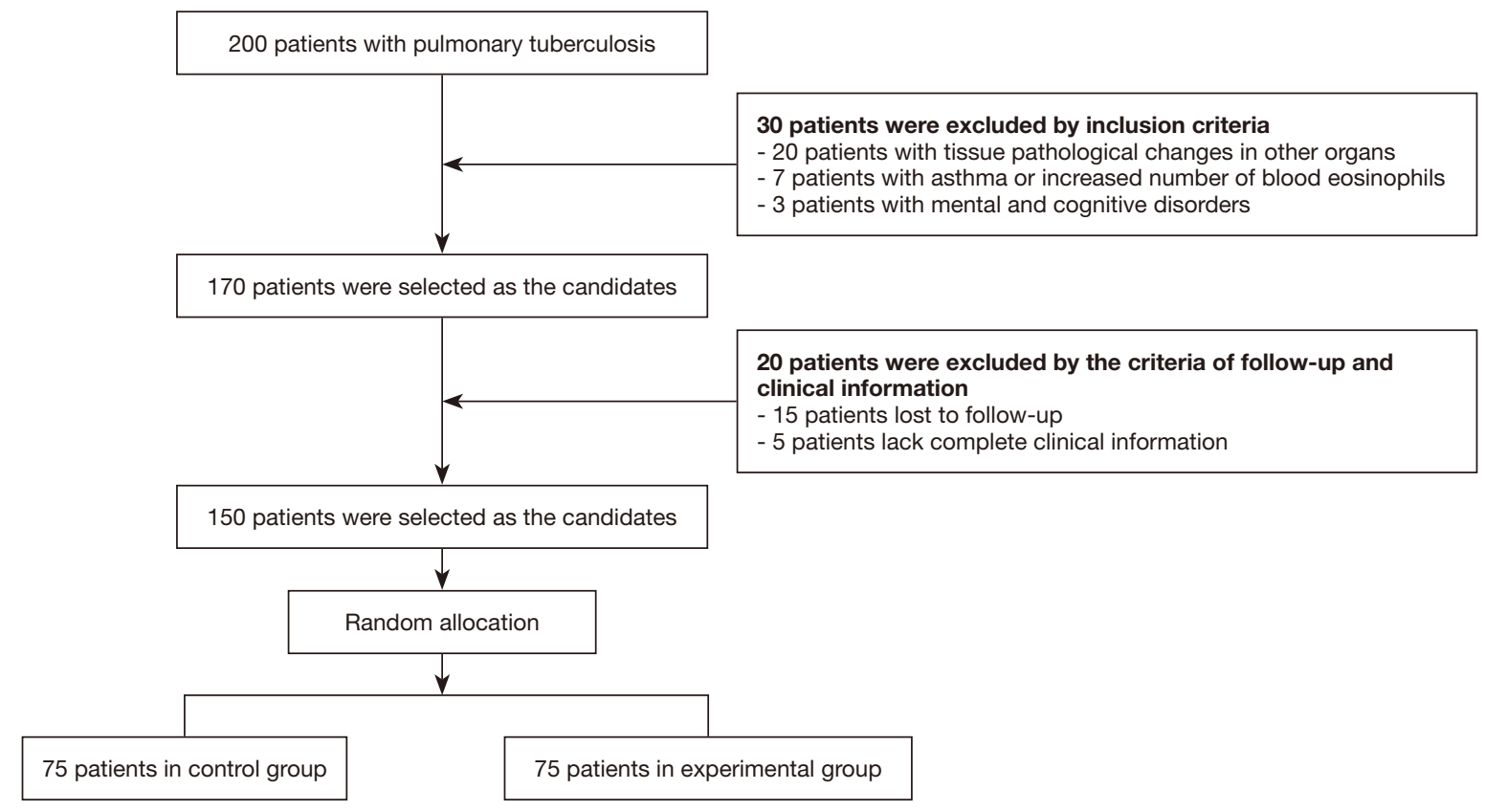

Figure 1 Study profile.

the use of multimedia audio and brochures. Enhancing the understanding and perception of a disease may help to improve a patient's treatment compliance and selfcontrol ability. Medication guidance was provided whereby medical staff informed patients and their families of the effects and precautions of drugs as well as the importance of drug intervention. Raising the patient's awareness of the medication used will improve prognosis and boost body immunity. Medical staff formulated scientific and rational dietary regimens according to the specific physiques of each patient and provided guidance in the form of a reasonable diet plan.

Patients in the experimental group were also given respiratory functional exercises. This involved sputum excretion training with the assistance of medical staff, and respiratory functional exercises such as pursed-lip breathing, abdominal breathing, squatting, as well as chestexpanding. During the process of rehabilitation, medical staff should encourage patients to exercise appropriately, such as practicing Tai Chi, slow walking, jogging, etc., with good control of the intensity and duration of exercise. In addition, systematic rehabilitation programs should be developed to progressively increase the exercise regimen (16-18).

\section{Observation indexes}

\section{Pulmonary function}

Spirometry was used to measure the indexes of pulmonary function including forced expiratory volume in one second (FEV1), forced vital capacity (FVC), and the ratio of FEV1 to FVC (FEV1/FVC). Differences between the two groups were compared and analyzed.

\section{The score of 6-minute walking distance (6MWD)}

The 6 MWD refers to the distance a patient can walk along a 1-meter round trip on a flat wide surface without shortness of breath over a 6-minute period. Patients can take a rest if they lack physical strength midway. The total walking distance after 6 minutes is then recorded.

\section{Evaluation of dyspnea using the modified Medical Research Council (mMRC) dyspnea scale}

The degree of dyspnea was evaluated before and after intervention on a scale graded from $0-5$. Patients who experienced dyspnea only during excessive or strenuous activities were graded 0 and scored 4 points. Patients who experienced transient tachypnea during fast walking on a flat road or walking on an incline were graded 1 and scored 
Table 1 A comparison of pulmonary function indexes between the experimental group and the control group ( \pm S)

\begin{tabular}{lccc}
\hline Group & FEV1 $(\mathrm{L})$ & FVC $(\mathrm{L})$ & FEV1/FVC $(\%)$ \\
\hline Experimental group $(\mathrm{n}=75)$ & $1.59 \pm 0.82$ & $2.45 \pm 0.78$ & $58.44 \pm 8.81$ \\
Control group $(\mathrm{n}=75)$ & $1.24 \pm 0.55$ & $1.83 \pm 0.87$ & $54.85 \pm 7.92$ \\
$\mathrm{t}$ & 3.0699 & 4.5952 & 2.6244 \\
$\mathrm{P}$ & 0.0025 & 0.000 & 0.0096 \\
\hline
\end{tabular}

FEV1, forced expiratory volume in one second; FVC, forced vital capacity.

3 points. Patients with lower walking speeds compared to their peers on a flat road or had to take a rest halfway were graded 2 and scored 2 points. Patients with tachypnea after walking a few minutes or about 100 meters on a flat road and had to take a rest were graded 3 and scored 1 point. Patients who experienced tachypnea while putting on or taking off clothes were graded 4 and scored 0 .

The partial pressure of blood oxygen $\left(\mathrm{PaO}_{2}\right)$ and partial pressure of carbon dioxide in the artery $\left(\mathrm{PaCO}_{2}\right)$

The $\mathrm{PaO}_{2}$ and $\mathrm{PaCO}_{2}$ were assessed in patients using bloodgas analyzers.

\section{Self-care ability}

The self-care ability of patients was evaluated by the exercise of self-care agency (ESCA) scale, including health knowledge level, self-care skills, sense of self-care responsibility, and self-concept. Higher scores indicated better self-care ability.

\section{Quality of life}

The quality of life of the patients after nursing intervention was evaluated using the health-related quality of life questionnaire, including questions related to daily activities, social activities, depression, and anxiety. Higher scores on daily activities and social activities indicated better quality of life, and higher scores on depression and anxiety indicated poorer psychological state.

\section{Statistical treatment}

Data was analyzed and represented using SPSS20.0 and GraphPad Prism 7 (GraphPad Software, San Diego, USA), respectively. Measurement data were compared using the Student's $t$-test and enumeration data were assessed using the $\chi^{2}$ test and normality test. A P value $<0.05$ was considered statistically significant.

\section{Results}

A comparison of pulmonary function indexes between the experimental group and the control group

After nursing intervention, all pulmonary function indexes of patients in the experimental group were significantly higher than those in the control group $(\mathrm{P}<0.05$; Table 1).

\section{A comparison of the 6MWD between the experimental group and the control group}

The 6MWD after nursing intervention in both groups was significantly higher than that before intervention. Furthermore, after intervention, the 6MWD in the experimental group was significantly higher than that in the control group $(\mathrm{P}<0.05$; Figure 2$)$.

\section{A comparison of the mMRC scale score between the experimental group and the control group}

The mMRC scores after nursing intervention in both groups were significantly higher than those before intervention. Furthermore, after intervention, the mMRC scores in the experimental group were significantly higher than those in the control group $(\mathrm{P}<0.05$; Figure 3$)$.

\section{$A$ Comparison of $\mathrm{PaO}_{2}$ and $\mathrm{PaCO}_{2}$ between the experimental group and the control group}

The $\mathrm{PaO}_{2}$ and $\mathrm{PaCO}_{2}$ in the experimental group were significantly better than those in the control group $(\mathrm{P}<0.05$; Figure 4).

\section{A comparison of self-care ability between the experimental group and the control group}

The scores of health knowledge level, self-care skills, 


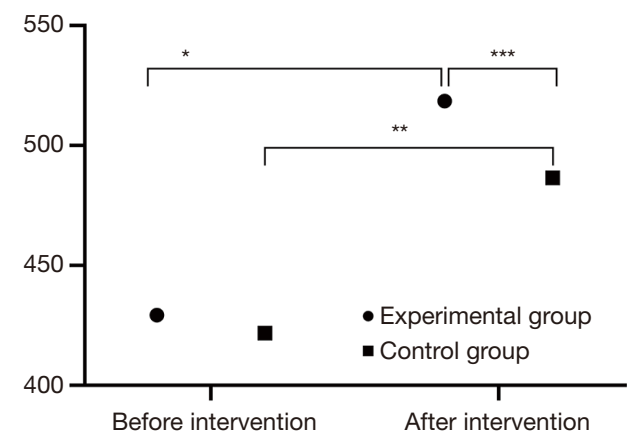

Figure 2 A comparison of the $6 \mathrm{MWD}$ between the experimental group and the control group $(\bar{x} \pm s)$. The abscissa represents before and after nursing intervention, while the ordinate represents distance $(\mathrm{m})$. In the experimental group, the 6MWD before and after nursing intervention were $429.3 \pm 51.7$ and $518.5 \pm 59.8 \mathrm{~m}$, respectively. In the control group, the $6 \mathrm{MWD}$ before and after nursing intervention were $421.8 \pm 52.5$ and $486.5 \pm 58.3 \mathrm{~m}$, respectively. *, indicates a significant difference in 6MWD before and after nursing intervention in the experimental group $(t=9.7722$, $\mathrm{P}=0.000) .{ }^{* *}$, indicates a significant difference in $6 \mathrm{MWD}$ before and after nursing intervention in the control group $(\mathrm{t}=7.1419$, $\mathrm{P}=0.000)$. ${ }^{* * *}$, indicates a significant difference in 6MWD between the two groups after nursing intervention $(\mathrm{t}=3.3183, \mathrm{P}=0.0011)$. 6MWD, 6-minute walking distance.

sense of self-care responsibility, and self-concept in the experimental group were significantly higher than those in the control group $(\mathrm{P}<0.001$; Table 2).

\section{A comparison of the quality of life between the experimental group and the control group}

The life quality scores in the experimental group were significantly better than those in the control group $(\mathrm{P}<0.001$; Table 3).

\section{Discussion}

Pulmonary tuberculosis is a disease with a high rate of recurrence and mortality. Clinically, many antagonistic drugs such as rifampicin and isoniazid have shown obvious therapeutic effects, but these drugs also have many adverse effects, leading to unsatisfactory outcomes (19-22). In recent years, many researchers have focused on respiratory rehabilitation nursing. Studies by Ngai et al. (23) on

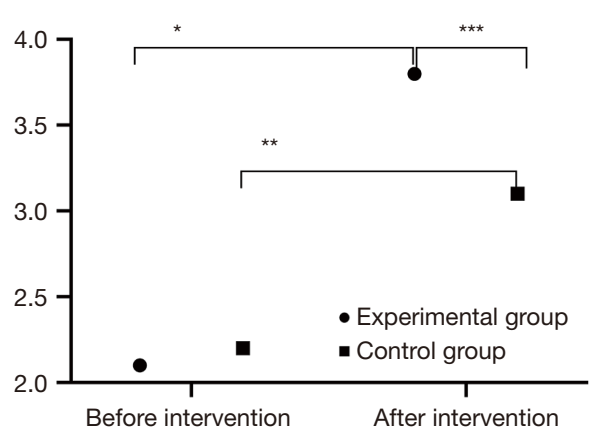

Figure 3 A comparison of $\mathrm{mMRC}$ between the experimental group and the control group $(\bar{x} \pm s)$. The abscissa represents before and after nursing intervention, while the ordinate represents $\mathrm{mMRC}$ score. In the experimental group, the $\mathrm{mMRC}$ scores before and after nursing intervention were $2.1 \pm 0.4$ and $3.8 \pm 0$.6, respectively. In the control group, the $\mathrm{mMRC}$ scores before and after nursing intervention were $2.2 \pm 0.5$ and $3.1 \pm 0.4$, respectively. ${ }^{*}$, indicates a significant difference in $\mathrm{mMRC}$ scores before and after nursing intervention in the experimental group $(\mathrm{t}=20.4163, \mathrm{P}=0.000)$. **, indicates a significant difference in $\mathrm{mMRC}$ scores before and after nursing intervention in the control group $(\mathrm{t}=12.1725, \mathrm{P}=0.000)$. ***, indicates a significant difference in mMRC scores between the two groups after nursing intervention $(\mathrm{t}=8.4067, \mathrm{P}=0.000)$. mMRC, modified Medical Research Council.

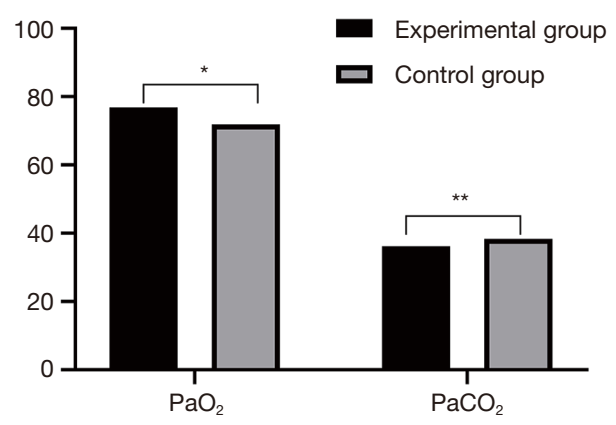

Figure $4 \mathrm{~A}$ comparison of $\mathrm{PaO}_{2}$ and $\mathrm{PaCO}_{2}$ between the experimental group and the control group $(\bar{x} \pm \mathrm{S})$. The abscissa represents $\mathrm{PaO}_{2}$ and $\mathrm{PaCO}_{2}$, while the ordinate represents $\mathrm{PaO}_{2}$ and $\mathrm{PaCO}_{2}$ values $(\mathrm{mmHg})$. In the experimental group, the $\mathrm{PaO}_{2}$ and $\mathrm{PaCO}_{2}$ were $76.9 \pm 4.5$ and $36.2 \pm 2.6 \mathrm{mmHg}$, respectively. In the control group, the $\mathrm{PaO}_{2}$ and $\mathrm{PaCO}_{2}$ were $71.9 \pm 3.4$ and $38.4 \pm 1.7 \mathrm{mmHg}$, respectively. *, indicates a significant difference in $\mathrm{PaO}_{2}$ between the two groups $(\mathrm{t}=7.6775, \mathrm{P}=0.000) .{ }^{* *}$, indicates a significant difference in $\mathrm{PaCO}_{2}$ between the two groups $(\mathrm{t}=6.1332$, $\mathrm{P}=0.000)$. 
Table 2 A comparison of self-care ability between the experimental group and the control group (s)

\begin{tabular}{lcccc}
\hline Group & Health knowledge level & Self-care skills & Sense of self-care responsibility & Self-concept \\
\hline Experimental group $(n=75)$ & $40.02 \pm 4.35$ & $45.66 \pm 3.12$ & $28.74 \pm 6.01$ & $28.49 \pm 3.78$ \\
Control group $(\mathrm{n}=75)$ & $31.24 \pm 4.42$ & $36.96 \pm 6.64$ & $21.13 \pm 6.38$ & $23.18 \pm 3.71$ \\
t & 12.2610 & 10.2698 & 7.5191 & 8.6824 \\
P & $<0.001$ & $<0.001$ & $<0.001$ & $<0.001$ \\
\hline
\end{tabular}

Table 3 A comparison of the quality life between the experimental group and the control group (s)

\begin{tabular}{lcccc}
\hline Group & Daily activities & Social activities & Depression psychology & Anxiety psychology \\
\hline Experimental group $(\mathrm{n}=75)$ & $44.9 \pm 9.2$ & $28.6 \pm 2.1$ & $19.4 \pm 0.5$ & $16.7 \pm 0.5$ \\
Control group $(\mathrm{n}=75)$ & $37.6 \pm 8.3$ & $25.9 \pm 1.8$ & $20.1 \pm 0.4$ & $21.7 \pm 0.8$ \\
$\mathrm{t}$ & 5.1022 & 8.4540 & 9.4675 & 48.8992 \\
$\mathrm{P}$ & $<0.001$ & $<0.001$ & $<0.001$ & $<0.001$ \\
\hline
\end{tabular}

respiratory rehabilitation nursing have demonstrated that scientific and reasonable respiratory functional exercises can significantly improve the pulmonary function of patients with pulmonary tuberculosis, enhance body endurance, and can further improve self-care ability. This concurs with the current study, which showed that after nursing intervention, the pulmonary function indexes and self-care ability in the experimental group were significantly higher than those in the control group. The scores of the 6MWD and $\mathrm{mMRC}$ in the experimental group were significantly higher after nursing intervention compared to before intervention. Furthermore, the scores in the experimental group after intervention were significantly higher than those in the control group after intervention. The $\mathrm{PaO}_{2}$, $\mathrm{PaCO}_{2}$, and quality of life in the experimental group after the intervention were significantly better than those in the control group. Comprehensive nursing intervention combined with respiratory functional exercises lay emphasis on improving the patient's understanding of pulmonary tuberculosis from a cognitive and psychological perspective, allowing them to fully recognize the importance of respiratory functional exercises for recovery and motivating their sense of self-care responsibility and confidence, thereby raising an awareness of health and improving selfcare ability.

In conclusion, comprehensive nursing intervention combined with respiratory functional exercises can improve the pulmonary function, self-care ability, and quality of life of patients with pulmonary tuberculosis. Enhancing a patient's understanding of the tuberculosis disease and stimulating their sense of responsibility for health management is a worthy clinical application and should be encouraged.

\section{Acknowledgments}

Funding: None.

\section{Footnote}

Reporting Checklist: The authors have completed the CONSORT reporting checklist. Available at https://dx.doi. org/10.21037/apm-21-1178

Trial Protocol: Available at https://dx.doi.org/10.21037/apm21-1178

Data Sharing Statement: Available at https://dx.doi. org/10.21037/apm-21-1178

Conflicts of Interest: All authors have completed the ICMJE uniform disclosure form (available at https://dx.doi. org/10.21037/apm-21-1178). The authors have no conflicts of interest to declare.

Ethical Statement: The authors are accountable for all aspects of the work in ensuring that questions related to the accuracy or integrity of any part of the work are 
appropriately investigated and resolved. This study was approved by the Ethics Committee of Wuhan Jinyintan Hospital (No.: 2016112505). All patients and their families were informed of the purpose and process of this study, received nursing intervention protocols, and signed the informed consent. The study followed the guild lines of Declaration of Helsinki (as revised in 2013).

Open Access Statement: This is an Open Access article distributed in accordance with the Creative Commons Attribution-NonCommercial-NoDerivs 4.0 International License (CC BY-NC-ND 4.0), which permits the noncommercial replication and distribution of the article with the strict proviso that no changes or edits are made and the original work is properly cited (including links to both the formal publication through the relevant DOI and the license). See: https://creativecommons.org/licenses/by-nc-nd/4.0/.

\section{References}

1. Sánchez-Cabral O, Santillán-Díaz C, Flores-Bello ÁP, et al. GeneXpert ${ }^{\circledR}$ MTB/RIF assay with transbronchial lung cryobiopsy for Mycobacterium tuberculosis diagnosis. Ann Transl Med 2020;8:351.

2. Hameed HMA, Tan Y, Islam MM, et al. Phenotypic and genotypic characterization of levofloxacin- and moxifloxacin-resistant Mycobacterium tuberculosis clinical isolates in southern China. J Thorac Dis 2019;11:4613-25.

3. Spitaleri A, Ghodousi A, Miotto P, Cirillo DM. Whole genome sequencing in Mycobacterium tuberculosis. Ann Transl Med 2019;7:S197.

4. Shi Q, Wang J, Yang Z, Liu Y. CircAGFG1modulates autophagy and apoptosis of macrophages infected by Mycobacterium tuberculosis via the Notch signaling pathway. Ann Transl Med 2020;8:645.

5. Caplan GA, Williams AJ, Daly B, et al. A randomized, controlled trial of comprehensive geriatric assessment and multidisciplinary intervention after discharge of elderly from the emergency department--the DEED II study. J Am Geriatr Soc 2004;52:1417-23.

6. Zhang $\mathrm{H}, \mathrm{Zhu} \mathrm{G}$, Chen J. The effects of comprehensive nursing interventions on sexual functions in young and middle-aged patients with coronary stent implantation. Health 2013;5:1-4.

7. Al Majid FM, Abba AA. Immunophenotypic characterisation of peripheral $\mathrm{T}$ lymphocytes in pulmonary tuberculosis. J Postgrad Med 2008;54:7-11.

8. Girdler-Brown BV, White NW, Ehrlich RI, et al. The burden of silicosis, pulmonary tuberculosis and COPD among former Basotho goldminers. Am J Ind Med 2008;51:640-7.

9. Williams E, Cheng AC, Lane GP, et al. Delays in presentation and diagnosis of pulmonary tuberculosis: a retrospective study of a tertiary health service in Western Melbourne, 2011-2014. Intern Med J 2018;48:184-93.

10. Bertazone Edo C, Gir E, Hayashida M. Nursing workers' experiences in care for pulmonary tuberculosis patients. Rev Lat Am Enfermagem 2005;13:374-81.

11. World Medical Association. World Medical Association Declaration of Helsinki: ethical principles for medical research involving human subjects. JAMA 2013;310:2191-4.

12. Petrović S. Diagnostic value of certain methods for isolation of Mycobacterium tuberculosis in children with suspected pulmonary tuberculosis. Med Pregl 2005;58:231-5.

13. Armijos RX, Weigel MM, Chacon R, et al. Adjunctive micronutrient supplementation for pulmonary tuberculosis. Salud Publica Mex 2010;52:185-9.

14. Singh J, Dalal P, Rattan KN. Congenital pulmonary airway malformation mimicking as pulmonary tuberculosis in five paediatric patients: a diagnostic dilemma. Trop Doct 2018;48:247-50.

15. Soto A, Solari L, Agapito J, et al. Algorithm for the diagnosis of smear-negative pulmonary tuberculosis in high-incidence resource-constrained settings. Trop Med Int Health 2013;18:1222-30.

16. Soto A, Solari L, Gotuzzo E, et al. Performance of an algorithm based on WHO recommendations for the diagnosis of smear-negative pulmonary tuberculosis in patients without HIV infection. Trop Med Int Health 2011;16:424-30.

17. Su MC, Hsieh YT, Wang YH, et al. Exercise capacity and pulmonary function in hospital workers recovered from severe acute respiratory syndrome. Respiration 2007;74:511-6.

18. Van Erck-Westergren E, Franklin SH, Bayly WM. Respiratory diseases and their effects on respiratory function and exercise capacity. Equine Vet J 2013;45:376-87.

19. Allen KJ, Terron-Canedo N, Hillyer MH, et al. Equitation and exercise factors affecting dynamic upper respiratory tract function: A review illustrated by case reports. Equine Veterinary Education 2011;23:361-8.

20. Morotomi N, Saitoh M, Takanashi S, et al. After omental flap transposition, respiratory function and exercise capacity decrease. Ann Thorac Cardiovasc Surg 
2010;16:9-15.

21. Ascensão AA, Magalhães JF, Soares JM, et al. Cardiac mitochondrial respiratory function and oxidative stress: the role of exercise. Int J Sports Med 2005;26:258-67.

22. Giannikoulis C, Karkoulias K, Thomopoulos K, et al. Patients with gastroesophageal reflux disease and respiratory manifestations do not present lung function disorders during cardiopulmonary exercise test. Dis

Cite this article as: $\mathrm{Xu} \mathrm{Z,} \mathrm{Chen} \mathrm{W,} \mathrm{Li} \mathrm{X.} \mathrm{Effects} \mathrm{of}$ comprehensive nursing intervention combined with respiratory functional exercises on pulmonary function and self-care ability in patients with pulmonary tuberculosis: results of a randomized trial. Ann Palliat Med 2021;10(7):7543-7550. doi: 10.21037/ apm-21-1178
Esophagus 2011;24:189-93.

23. Ngai JC, Ko FW, Ng SS, et al. The long-term impact of severe acute respiratory syndrome on pulmonary function, exercise capacity and health status. Respirology 2010;15:543-50.

(English Language Editor: J. Teoh) 